\title{
Impact of resistance to first-line and injectable drugs on treatment outcomes
} in MDR-TB

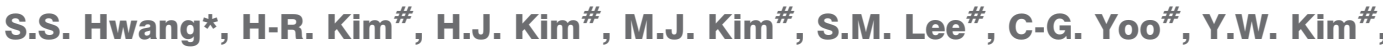 \\ S.K. Han ${ }^{\#}$, Y-S. Shim ${ }^{\#}$ and J-J. Yim ${ }^{\#}$
}

ABSTRACT: Recently, resistance to additional first-line and injectable drugs was reported to be an independent risk factor for adverse outcomes in multidrug-resistant (MDR) tuberculosis (TB) patients. The aim of the present study was to confirm these observations in MDR-TB patients without HIV infection.

MDR-TB patients treated at a tertiary referral hospital in South Korea between January 1996 and December 2005 were included. The unadjusted and adjusted odds ratios of adverse treatment outcome were calculated for resistance to each drug and combination of drugs using simple or multiple logistic regressions.

None of the resistance to additional first-line or injectable drugs was associated with higher odds for adverse treatment outcome in 155 MDR but nonextensively drug-resistant (non-XDR) TB patients. However, streptomycin resistance was associated with 12 times the odds for adverse treatment outcome in 42 extensively drug-resistant (XDR) TB patients. Neither combinations of first-line drugs nor those of injectable drugs were associated with increased odds for adverse treatment outcomes in non-XDR MDR-TB patients or XDR-TB patients.

Only streptomycin resistance among the first-line or injectable drugs was associated with adverse treatment outcomes in extensively drug-resistant tuberculosis patients without HIV infection.

KEYWORDS: Extensive drug resistance, multidrug resistance, streptomycin, tuberculosis

$\mathbf{R}$ ecently, concerns regarding extensively drug-resistant (XDR) tuberculosis (TB), which shows extensive resistance to second-line anti-TB drugs in addition to isoniazid and rifampicin, have been raised [1-3]. According to a 2005 survey that included 25 reference laboratories on six continents, $\sim 10 \%$ of all multidrug-resistant (MDR) TB strains were XDR-TB [2]. Among XDR-TB patients with HIV infection, an alarmingly high fatality rate of nearly $100 \%$, with a median survival of only 16 days, was reported [4]. However, XDR-TB has been reported not only among HIV-infected populations, but also among non-HIV-infected patients $[5,6]$. The current authors recently examined 211 MDR-TB patients without HIV infection in South Korea and reported that the presence of XDR-TB posed 4.46 times higher odds of treatment failure [7]. This observation was confirmed in European patients with MDR-TB [8] and additional Korean MDR-TB cohorts [9, 10].

Recent studies have also suggested that in addition to XDR-TB, resistance to additional first-line drugs [8], kanamycin [9] and capreomycin [11], are independent risk factors for adverse treatment outcomes. However, this observation has not been confirmed in other MDR-TB cohorts. The aim of the present study was to confirm the impact of resistance to additional first-line and injectable drugs on treatment outcomes in MDRTB patients without HIV infection in a South Korean population.

\section{METHODS}

\section{Inclusion criteria}

The current study was a retrospective cohort study based on the reanalysis of a previously published data set, which included 211 patients with MDR-TB [7]. All patients were treated at Seoul National University Hospital (Seoul, Republic of Korea), a university-affiliated tertiary referral hospital, between January 1996 and December 2005. After excluding seven defaulted and seven transferred-out patients, 197 MDR-TB patients were included in the final analyses. The Ethical Review Committee of the Seoul National University Hospital approved the current study.

\section{AFFILIATIONS}

${ }^{*}$ Dept of Social and Preventive Medicine, College of Medicine, Inha University, Incheon, and "Division of Pulmonary and Critical Care Medicine, Dept of Internal Medicine and Lung Institute, Seoul National University College of Medicine, Seoul, Republic of Korea.

CORRESPONDENCE

J-J. Yim

Division of Pulmonary and Critical Care Medicine

Dept of Internal Medicine

Seoul National University College of Medicine

103 Daehangno Jongno-gu Seoul 110-744

Republic of Korea

Fax: 82220729662

E-mail: yimjj@snu.ac.kr

Received:

July 012008

Accepted after revision:

October 202008

\section{SUPPORT STATEMENT}

The present study was funded by grant 04-2006-115-0 from the Seoul National University College of Medicine Research Fund (Seoul, Republic of Korea).

STATEMENT OF INTEREST None declared. 


\section{Mycobacterial culture and drug susceptibility tests}

Mycobacterial cultures were performed in 3\% Ogawa medium at the Seoul National University Hospital. Drug susceptibility tests (DST) were performed at the Korean Institute of Tuberculosis (Seoul), the supranational TB reference laboratory in South Korea. All samples were screened using proportional methods for isoniazid, rifampicin, ethambutol, streptomycin, kanamycin, enviomycin, ofloxacin, para-aminosalicylic acid and prothionamide sensitivity. Additionally, pyrazinamide sensitivity was determined using the pyrazinamidase test [12]. Tests for enviomycin resistance were replaced by tests for capreomycin resistance at the same concentration beginning in March 1996.

\section{Treatment for patients with MDR-TB}

Although treatment for each patient was individualised by the physician based on the DST results, the principles of treatment for patients with MDR-TB at the present institution were as follows: 1) use of any first-line agent showing susceptibility; 2) use of injectable anti-TB drugs and quinolones, if susceptible; 3) addition of second-line bacteriostatic agents as needed to complete the five-drug regimen; and 4) treatment for $2 \mathrm{yrs}$ after culture conversion. In addition, the criteria for surgical resection were MDR-TB refractory to $\geqslant 6$ months of medical treatment with a primary localised lesion [13].

\section{Definition of MDR- and XDR-TB}

MDR-TB was defined as TB caused by bacilli that showed resistance to at least isoniazid and rifampicin. XDR-TB was diagnosed based on a revised definition [14]. Since the current authors performed DST only for ofloxacin among the quinolone family and streptomycin, kanamycin and capreomycin/enviomycin among the injectable antibiotics, XDR-TB was defined as TB caused by bacilli that showed resistance to isoniazid, rifampicin and ofloxacin, and showed kanamycin or capreomycin/enviomycin resistance.

\section{Classification of treatment outcomes}

The treatment outcomes were classified into the following groups in accordance with the suggested criteria of LASERSON et al. [15]: cure; treatment completed; death; default; failure; and transferred out. In addition, if patients were diagnosed as having bacteriologically confirmed MDR-TB after being cured or after treatment was completed, they were classified as relapsed cases.

Based on these classifications, the treatment outcomes were further categorised into treatment success (for cured and treatment completed) or adverse treatment outcome (for death, failure and relapsed) to identify predictors of poor treatment response.

\section{Statistical analyses}

To assess the impact of first-line and injectable drug resistance, the odds ratios (ORs) of adverse treatment outcomes for each drug were calculated. Adjusted ORs were calculated after adjusting for sex, age, body mass index, presence of comorbidities, albumin level (as a continuous variable), surgical resection and the number of drugs used. In addition, the unadjusted and adjusted ORs of resistance to various combinations of first-line and injectable drugs were calculated. Unadjusted and adjusted ORs were calculated using simple or multiple logistic regressions and exact logistic regression when the estimate of the coefficient was zero or extreme, to obtain a maximum unbiased estimate.

\section{RESULTS}

\section{Demographic characteristics and treatment outcomes}

The median (range) age of the 197 patients with MDR-TB was 37 (13-91) yrs; 115 (58.4\%) patients were male. The median number of drugs to which Mycobacterium tuberculosis isolates were resistant was four (2-11). A median of six anti-TB drugs (3-12) were used for a median of 27 (1-136) months. Among the patients, $42(21.3 \%)$ had XDR-TB. Adverse treatment outcomes were more frequently observed in XDR-TB patients (45.2 versus $29.7 \%$; $\mathrm{p}=0.057$ ).

\section{Resistance to individual first-line and injectable drugs and treatment outcomes}

No resistance to first-line or injectable drugs (amikacin was excluded because DST was not performed for this agent) was associated with higher odds for an adverse treatment outcome among the 155 non-XDR MDR-TB patients. However, streptomycin resistance was associated with adverse treatment outcomes in 42 XDR-TB patients (adjusted OR 12.05, 95\% confidence interval (CI) 1.48-98.38). Excluding streptomycin, resistance to the other individual drugs was not associated with treatment outcome in XDR-TB patients (table 1).

\section{Impact of combinations of resistance on first-line and injectable drugs}

Neither combinations of first-line drugs nor those of injectable drugs were associated with any increased odds for adverse treatment outcomes in non-XDR MDR-TB patients or in XDRTB patients (table 2).

\section{DISCUSSION}

In addition to XDR-TB, resistance to additional first-line and injectable drugs has been reported to be associated with poor treatment outcomes among MDR-TB patients. Specifically, resistance to all additional first-line drugs was associated with a 2.61 times higher risk for an unfavourable outcome (death or failure) compared with MDR-TB patients susceptible to at least one additional first-line drug [8]. Capreomycin resistance was associated with a 3.51 times higher risk for unfavourable outcomes in European patients with MDR-TB [11]. In addition, kanamycin resistance had a 3.9 times higher risk for 6-month culture positivity in Korean patients [9].

The present study was not in agreement with previous reports of poor prognostic factors associated with first-line drugs or kanamycin/capreomycin resistance. Instead, the current results revealed the clinical significance of streptomycin resistance. Streptomycin resistance was associated with increased odds of adverse outcome among XDR-TB patients (adjusted OR 12.05, 95\% CI 1.48-98.38).

The fact that four recent studies, including the present one, have not found consistent evidence for the use of specific firstline and injectable drug resistances in predicting treatment outcomes (other than the presence of XDR-TB $[8,9,11]$ ) suggests a need for further studies prospectively enrolling larger numbers of MDR-TB patients to better define the clinical significance of drug resistance. However, the results of the current and previous studies are consistent in showing the 


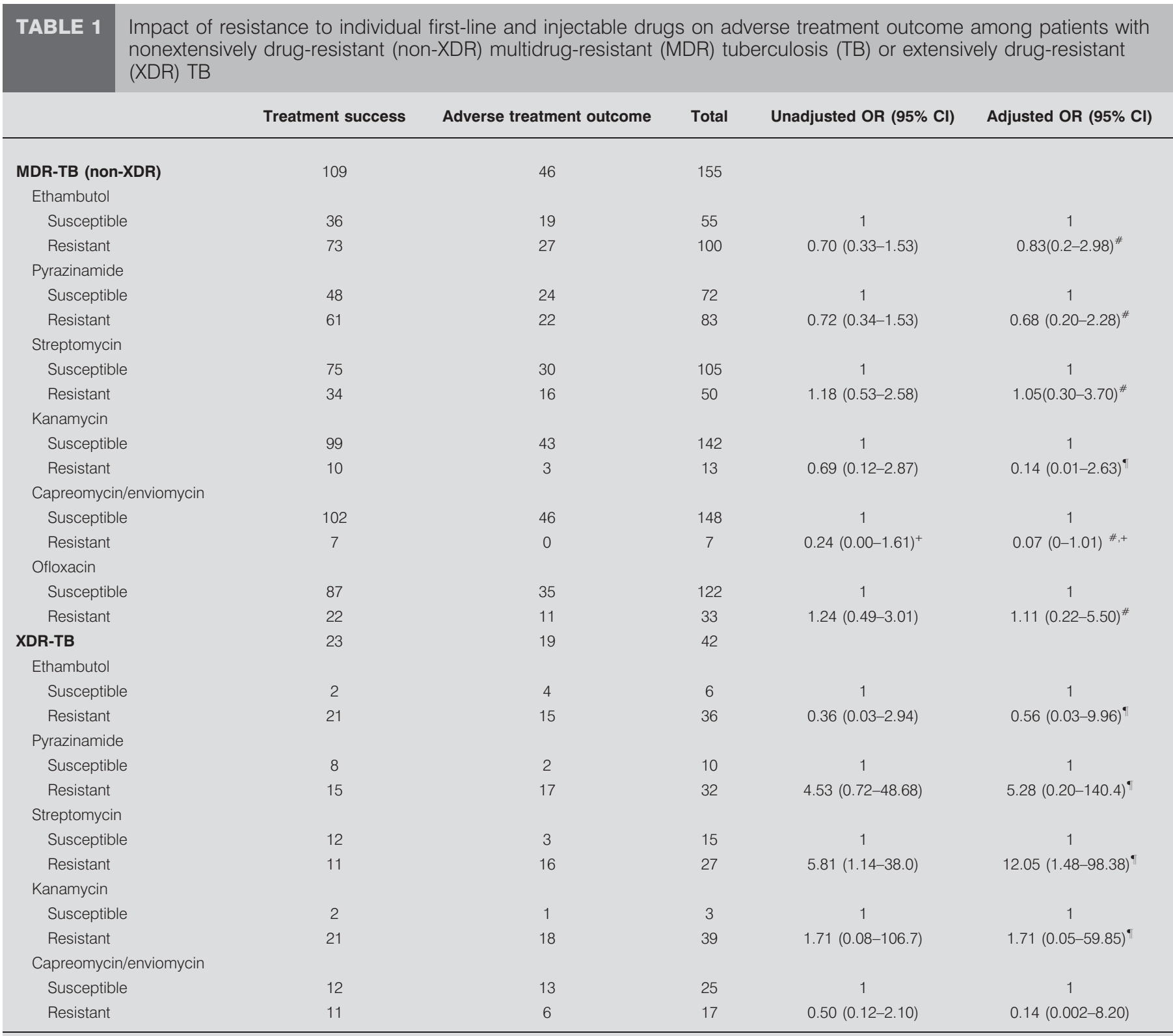

Data are presented as $\mathrm{n}$, unless otherwise stated. OR: odds ratio; Cl: confidence interval. * : adjusted for age, sex, body mass index (BMI), the presence of comorbidities, bilateral cavities, albumin levels, surgery and number of used drugs; ": adjusted for age, sex, the presence of comorbidities, bilateral cavities, albumin levels, surgery and number of used drugs, but not for BMI due to complete determination cases; ${ }^{+}$: OR was estimated using the exact logistic regression model.

importance of injectable drug resistance on treatment outcome. Kanamycin [9], capreomycin [11] and streptomycin resistance is associated with poor treatment outcomes. These results underscore the important role of injectables in MDR-TB or XDR-TB treatment. Lack of association between kanamycin/capreomycin and adverse treatment outcomes in the current study despite similar in vitro antimycobacterial activities of injectable drugs $[16,17]$ could be understood by the fact that the majority $(92.9 \%)$ of XDR-TB patients had kanamycin resistance and the irregular availability of capreomycin during the present study period in South Korea. Future studies with better design may be able to confirm the suggested important role of resistance to injectables in non-XDR MDR-TB or MDR-TB patients.
To fully appreciate these results, the strengths and weaknesses of the present study must be considered. First, only 14 patients defaulted or transferred out of treatment among the $221 \mathrm{MDR}-$ TB patients screened for the current study. In this context, the present study results are not subject to noncomparability from unequal attrition during follow-up. In addition, the fact that every DST was performed in a single supranational TB reference laboratory is likely to have minimised the differential misclassification of drug resistance. However, the current study also has several weaknesses. Susceptibility tests for quinolones (other than ofloxacin) and tests for amikacin were not performed. Subsequently, MDR-TB with the other quinolones or amikacin resistance could not be classified as XDR-TB, 


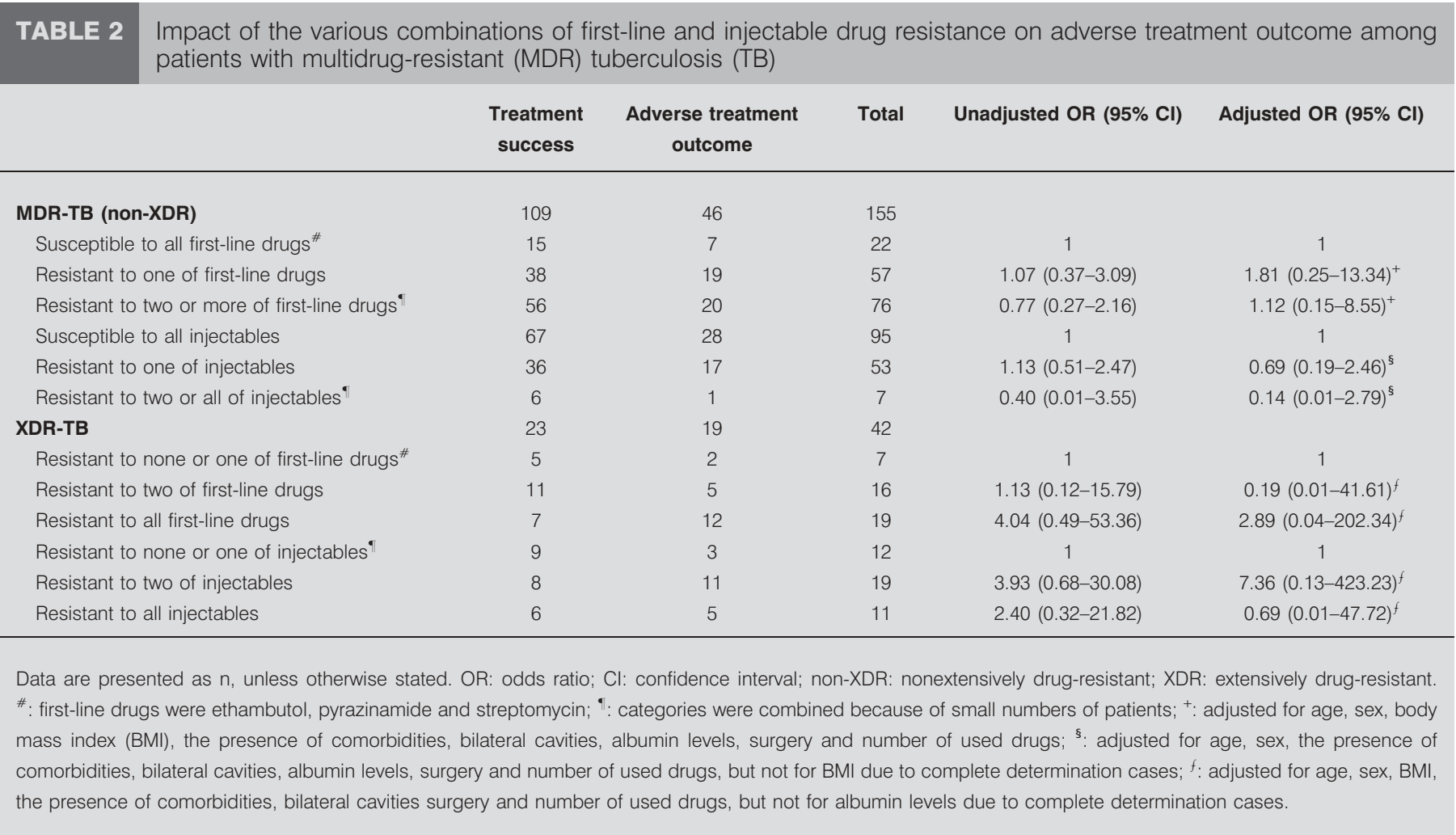

resulting in an underestimation of the proportion of XDR-TB among MDR-TB patients. In this context, it is possible that the clinical significance of streptomycin resistance may have been either over- or underestimated. Likewise, resistance to any of the fluoroquinolones may be associated with adverse treatment outcomes, despite a lack of association in the present study. Another weakness was the small number $(n=42)$ of XDR-TB patients. Although comparable to the numbers of XDR-TB patients in other studies, the small number of patients inevitably results in wide confidence intervals and leaves the possibility of insufficient statistical power to detect real differences between groups.

In conclusion, the current authors could not replicate the previously suggested association between resistance to first-line drugs, kanamycin and capreomycin, with adverse treatment outcomes in nonextensively drug-resistant multidrug-resistant tuberculosis patients or extensively drug-resistant tuberculosis patients without HIV infection in a South Korean population. Instead, only streptomycin resistance was associated with adverse treatment outcomes in extensively drug-resistant tuberculosis patients without HIV infection.

\section{REFERENCES}

1 Dukes Hamilton C, Sterling TR, Blumberg HM, et al. Extensively drug-resistant tuberculosis: are we learning from history or repeating it? Clin Infect Dis 2007; 45: 338-342.

2 Centers for Disease Control and Prevention (CDC). Emergence of Mycobacterium tuberculosis with extensive resistance to second-line drugs-worldwide, 2000-2004. MMWR Morb Mortal Wkly Rep 2006; 55: 301-305.

3 Raviglione M. XDR-TB: entering the post-antibiotic era? Int J Tuberc Lung Dis 2006; 10: 1185-1187.

4 Gandhi NR, Moll A, Sturm AW, et al. Extensively drugresistant tuberculosis as a cause of death in patients coinfected with tuberculosis and HIV in a rural area of South Africa. Lancet 2006; 368: 1575-1580.

5 Blaas SH, Mütterlein R, Weig J, et al. Extensively drug resistant tuberculosis in a high income country: a report of four unrelated cases. BMC Infect Dis 2008; 8: 60.

$6 \mathrm{Yu} \mathrm{MC}, \mathrm{Wu} \mathrm{MH}$, Jou R. Extensively drug-resistant tuberculosis, Taiwan. Emerg Infect Dis 2008; 14: 849-850.

7 Kim HR, Hwang SS, Kim HJ, et al. Impact of extensive drug resistance on treatment outcomes in non-HIVinfected patients with multidrug-resistant tuberculosis. Clin Infect Dis 2007; 45: 1290-1295.

8 Migliori GB, Besozzi G, Girardi E, et al. Clinical and operational value of the extensively drug-resistant tuberculosis definition. Eur Respir J 2007; 30: 623-626.

9 Jeon CY, Hwang SH, Min JH, et al. Extensively drugresistant tuberculosis in South Korea: risk factors and treatment outcomes among patients at a tertiary referral hospital. Clin Infect Dis 2008; 46: 42-49.

10 Kim DH, Kim HJ, Park SK, et al. Treatment outcomes and long-term survival in patients with extensively drugresistant tuberculosis. Am J Respir Crit Care Med 2008; 178: 1075-1082.

11 Migliori GB, Lange C, Centis R, et al. Resistance to second-line injectables and treatment outcomes in multidrug-resistant 
and extensively drug-resistant tuberculosis cases. Eur Respir J 2008; 31: 1155-1159.

12 Wayne LG. Simple pyrazinamidase and urease tests for routine identification of mycobacteria. Am Rev Respir Dis 1974; 109: 147-151.

13 Kim HJ, Kang CH, Kim YT, et al. Prognostic factors for surgical resection in patients with multidrug-resistant tuberculosis. Eur Respir J 2006; 28: 576-580.

14 World Health Organization. Extensively drug-resistant tuberculosis (XDR-TB): recommendations for prevention and control. Wkly Epidemiol Rec 2006; 81: 430-432.
15 Laserson KF, Thorpe LE, Leimane V, et al. Speaking the same language: treatment outcome definitions for multidrug-resistant tuberculosis. Int J Tuberc Lung Dis 2005; 9: 640-645.

16 Ho YI, Chan CY, Cheng AF. In-vitro activities of aminoglycoside-aminocyclitols against mycobacteria. J Antimicrob Chemother 1997; 40: 27-32.

17 Heifets L, Lindholm-Levy P. Comparison of bactericidal activities of streptomycin, amikacin, kanamycin, and capreomycin against Mycobacterium avium and $M$. tuberculosis. Antimicrob Agents Chemother 1989; 33: 1298-1301. 